\title{
Analysis of X-ray Patterns in Infection and Tumor
}

\author{
Dominic Puthoor ${ }^{1}$
}

\section{Abstract}

In diagnosis of bone tumor, X-ray is as important, if not more important than pathology report. However, X-ray had specific disadvantage. Tumor mimics especially infection can have same appearance as tumor in the X-ray. In this article, author analyzes the different patterns of X-ray changes common to tumor and infection and points outs that X-ray depicts the aggressiveness of the condition rather than a specific diagnosis.

Keywords: X-ray, Bone tumor, Tumor mimics

\section{Introduction}

Inflammation is body's reaction to anything detrimental to it. It may be trauma, microorganism, or tumor. Inflammation is the essential first step of healing $[1,2]$. Reaction of the body depends on the site rather than the cause. Whether it is infection, trauma, or tumor, the reaction is very much similar. On the other hand, inflammatory response widely varies with different tissues. This aspect is quite obvious in the bone where inflammatory reaction is totally different from that in soft tissue. Bone being a rigid structure, cannot swell. It lacks many aspects of inflammation common to other tissues.

What is the inflammatory change specific to the bone? How does bone reacts to infection, tumor, and trauma? Bone reacts with new bone formation because, it is how bone heals [3]. By new bone formation, body is trying to wall off the offender. How thick and effective the wall, depends on relative strength of the offender and defender. Primitive mesenchymal cell is recruited to become osteoblast that produced new bone. The new bone that is formed is woven bone irrespective of whether it is fractures, infection, or tumor (Woven bone because it is formed quickly).

Fig. 1 shows a case of incomplete fracture. Body reacted with new bone formation. The patient consulted an orthopedic surgeon who, possibly not taking history properly, made a diagnosis of osteosarcoma and referred the case to an oncology center. Because Xray picture was very much similar to osteosarcoma. Even pathological changes are so similar that, pathologist may report it as osteosarcoma if biopsy was taken. X-ray and computed tomography (CT) need not distinguish between infection, tumor, or trauma.

Will magnetic resonance imaging (MRI) help? The penumbra sign consisting of the central abscess cavity, the hyperintense granulation layer, the low signal intensity sclerotic bone reaction, and the surrounding marrow edema on T1-weighted MR images without contrast which has been shown to be a useful, though not a pathognomonic finding in subacute osteomyelitis [4].

What about blood investigations? Increase in total count, raised erythrocyte sedimentation rate (ESR) and C-reactive protein, though considered as indication of infection can occur to a lesser extend in tumors and trauma. This is because all these values indicate inflammatory response and not specific to the cause. Similarly, raised alkaline phosphatase and increased uptake in bone scan indicate an increase in osteoblastic activity and new bone formation and can occur not only in fracture but also in infection and tumors. This is because in all these conditions, body reacts with new bone formation. Then, how can you distinguish tumor and infection? What is the essential difference between infection and tumor? In tumor, cause is one's owns cells while in infection it is a different organism. Infection is like a foreign country attacking our nation, for example, China attacking India. Tumor is like terrorist, for example, Maoist who is an Indian citizen. In infection, body can promptly react. In tumor, body is at a loss because culprit is its own cells. This difference will be reflected in clinical picture, blood investigations and to a lesser extend in X-ray, CT, MRI, and bone scan [5]. It is biopsy that clearly distinguishes these two conditions. In histopathology, infection shows only inflammatory cells while tumor shows specific tumor cells. It is quite unusual to demonstrate bacterial organisms in tissues from patients with osteomyelitis. This is because the organisms are very small and a fairly high concentration of bacteria must be present for detection. Confirmation of diagnosis of infection is by culturing the organism.

Let us think more about X-ray changes in infection and tumors. X-ray depicts what tumor does to the bone and what bone does to tumor. Situation is not different in infection. In both, X-ray shows the sequelae of the fight between bone and infecting organism/tumor. For example, scalloping on the inner border of thickened cortex is classically described in chondrosarcoma [6] (Figs. 2a and b). Now

${ }^{1}$ Department of Orthopaedic Oncologist, Amala Institute of Medical Sciences, Thrissur, Kerala, India.

Address of Correspondence

Dr. Dominic Puthoor,

Department of Orthopaedic Oncologist, Amala Institute of Medical Sciences, Thrissur, Kerala, India.

E-mail: dkputhur@gmail.com

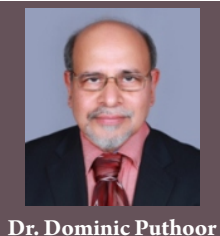

Submitted: 8/3/ 2021; Review: 26/3/ 2021; Accepted: 13/4/ 20201; Published: 10/5/ 2021

(C) 2021 by Journal of Bone and Soft Tissue Tumors | Available on www.jbstjournal.com| DOI:10.13107/jbst.2021.v07i02.47

This is an Open Access aticle distributed under the terms of the Creatiive Commons Attbution Non-Commercial-Share Alike 4.0 License (http:/eativecommons.org/licenses/by-nc-sa/4.0) which allows others to remix, tweak, and build upon the work non-commercially as long as appropriate credit is given and the new creation are licensed under the identical terms. 

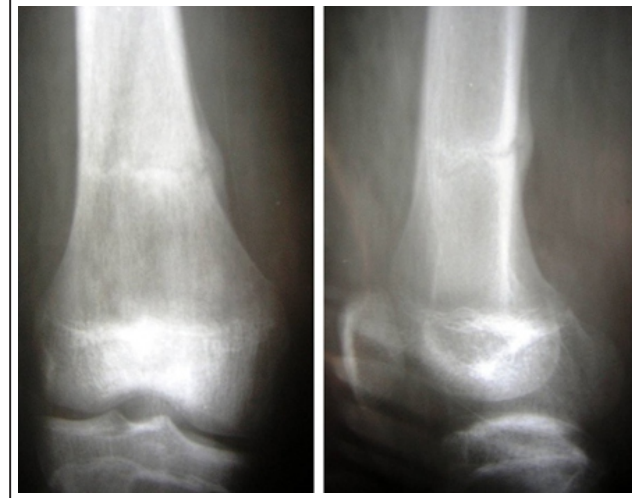

Figure 1: Incomplete fracture mimicking osteosarcoma.
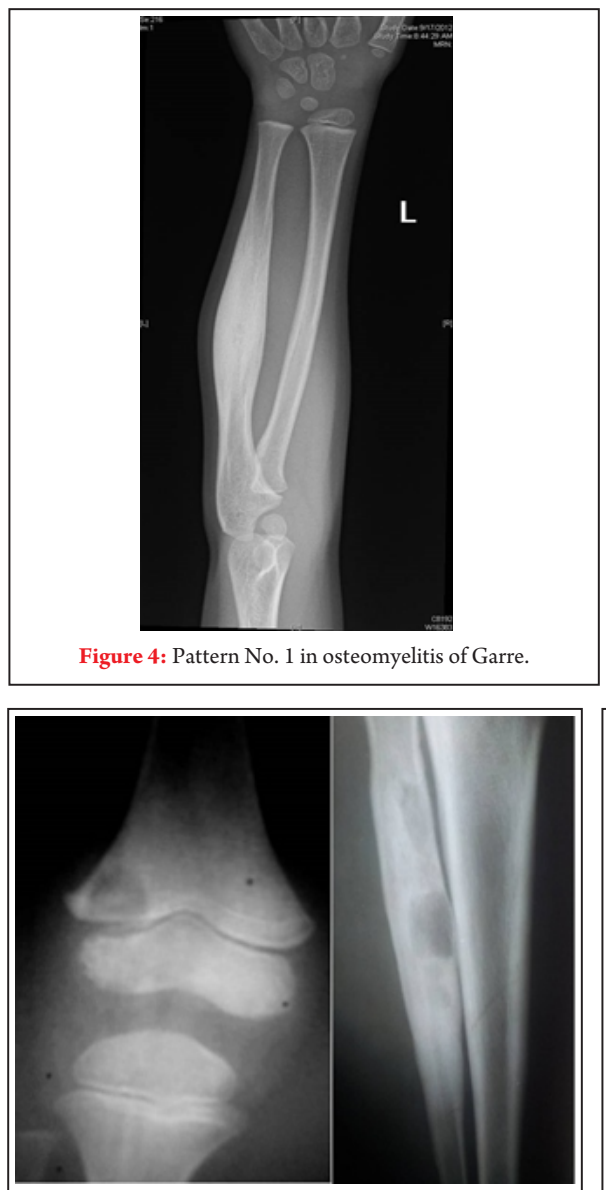

Figure 7: Pattern No. 2 in chronic osteomyelitis

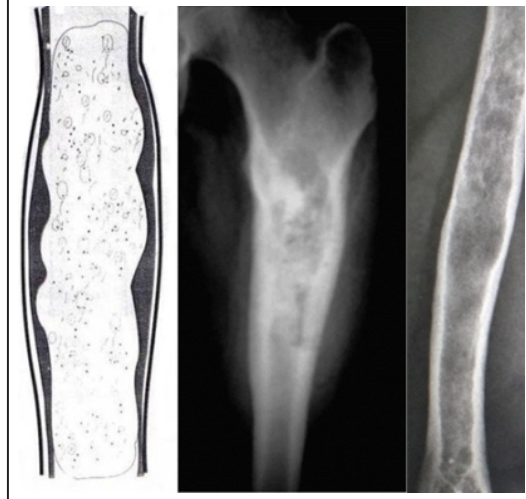

Figure 2: Endosteal scalloping in tumor and infection.

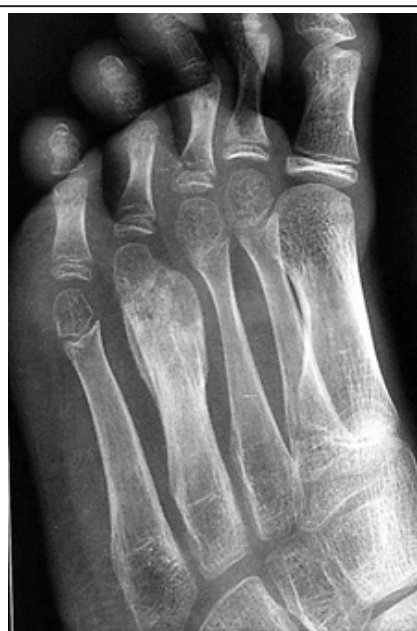

Figure 5: Pattern No. 1 in stress fracture in child.

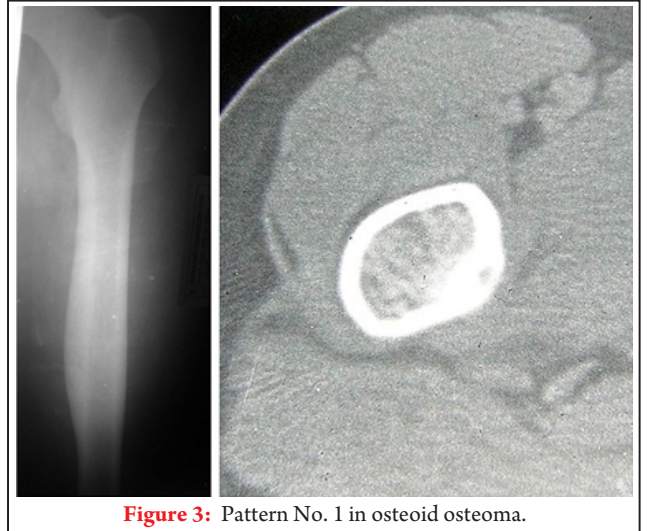

Figure 3: Pattern No. 1 in osteoid osteoma.

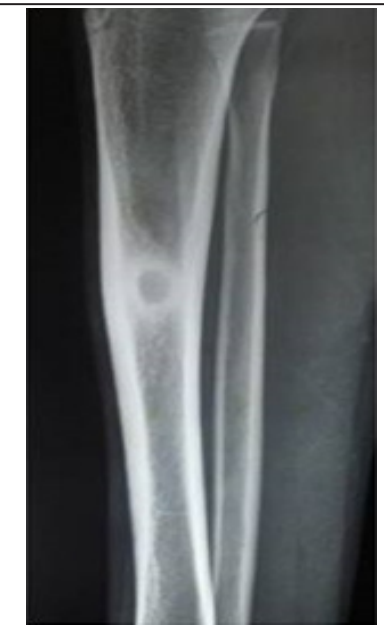

Figure 6: Pattern No. 2 in chronic osteomyelitis.

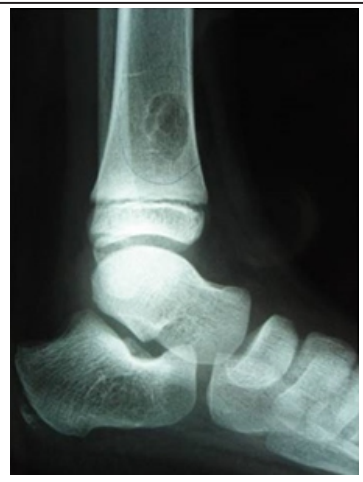

Figure 9: Pattern No. 2 in tumors

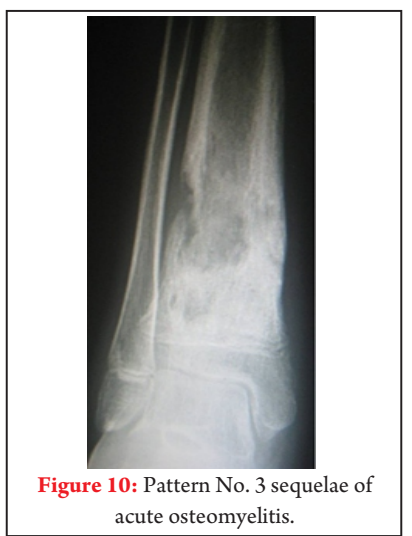

look at Fig. 2c. It is a case of chronic osteomyelitis presented with acute exacerbation. It also shows endosteal scalloping. That means what the offender is, do not matter much. Whether it is microorganism or tumor cells, picture is the same. Instead, who wins, whether it is bone (body) or microorganism/tumor, determines the $\mathrm{X}$-raypicture.

Depending on this concept, we can identify three distinct X-ray patterns [7].

\section{Pattern No 1}

Here, body (bone) has an upper hand and tumor/infecting organism is at a loss. Fig. 3a shows X-ray of a case of osteoid osteoma. Diagnosis of osteoid osteoma was done because of classical symptoms rather than the radiological picture. In X-ray, extensive solid periosteal reaction is the striking feature. Nidus is demonstrable in CT scan only (Fig. 3b).

Fig. 4 shows X-ray of a boy presented with bony swelling, pain increase on activities, and better relieved by antibiotics than nonsteroidal anti-inflammatory drugs. This is the classical picture of osteomyelitis of Garre. Here also, there is extensive solid periosteal reaction.

One is tumor and other is infection. Difference is in clinical presentation and peroperative findings. X-ray picture is similar. Solid periosteal reaction and extensive sclerosis disproportionate to the size of the lesion are the distinctive features of pattern No. 1. It indicates that body (bone) will inevitably conquer the offender whether it is tumor or infection. Still, it will be nice if you remove the offender with radiofrequency ablation in the first case and 


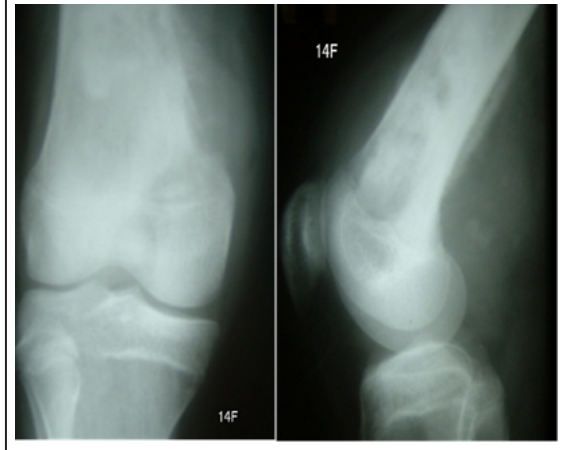

Figure 11: Pattern No. 3 in osteosarcoma

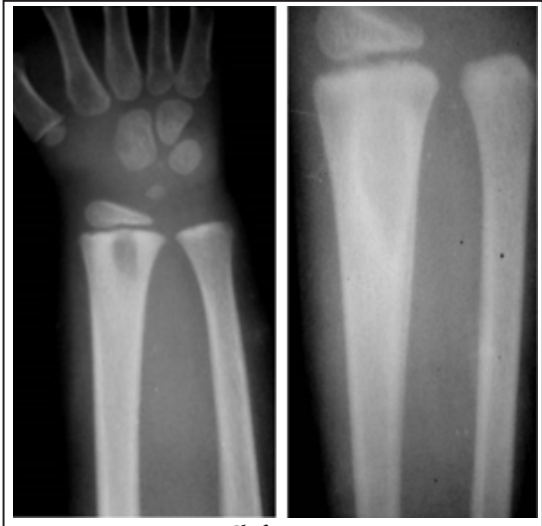

Figure 15: Shift in pattern 2 to 1

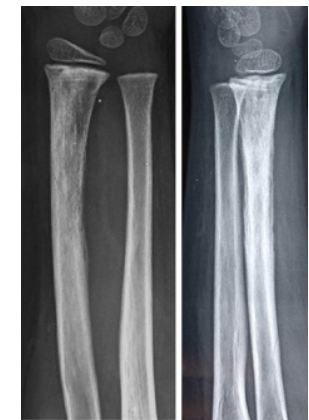

Figure 12: Pattern No. 3 in Ewing's sarcoma.

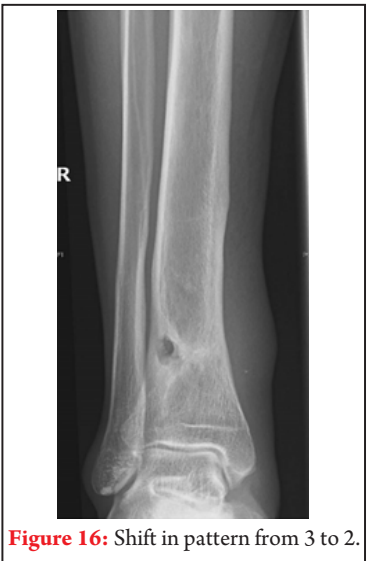

Figure 16: Shift in pattern from 3 to 2

saucerization in the second. Pattern No.1 occur in stress fracture also; especially in children (Fig. 5). This shows how the body wins over the pathological changes caused by repeated stress.

\section{Pattern No. 2}

Here, body (bone) and tumor/infecting organism are of equal strength. Fig. 6 shows X-ray of a 16F presented with painful swelling leg of 1 year's duration. On examination, there is a tender hard swelling. Overlying skin is normal, there is local raise of temperature. ESR: 74, CRP: 16.2. Peroperatively, there was about $1 \mathrm{~mL}$ of pus with dirty granulation tissue filling up the lesion.

Both Fig. 7a and b also show X-rays of cases of infection. Here, bone is reacting but bacteria are not yielding necessitating intervention of surgeon.

Now let us consider tumors. Fig. 8 is a case of chondroblastoma in its classical locations. Fig. 9 is eosinophilic granuloma. Here also, bone is defending but ultimately has to yield. In cases of benign aggressive tumors, body's reaction, hence, the X-ray picture is similar to chronic osteomyelitis. In contrast to Pattern 1, in Pattern 2, lesion is bigger. Although there is a clear margin, surrounding sclerosis is not extensive. This means whether it is infection or tumor, unless orthopedic surgeon comes for help, lesion may destroy the bone and ultimately the body.

\section{Pattern No. 3}

Here, tumor/infecting organism is very powerful and body (bone) is at a loss. Fig. 10 shows X-ray of a case of acute osteomyelitis who initially presented with classical clinical features. However, drainage was delayed. See the devastating damage it had caused.

Fig. 11 is a case of osteosarcoma. Here, body is totally at a loss. Culprit
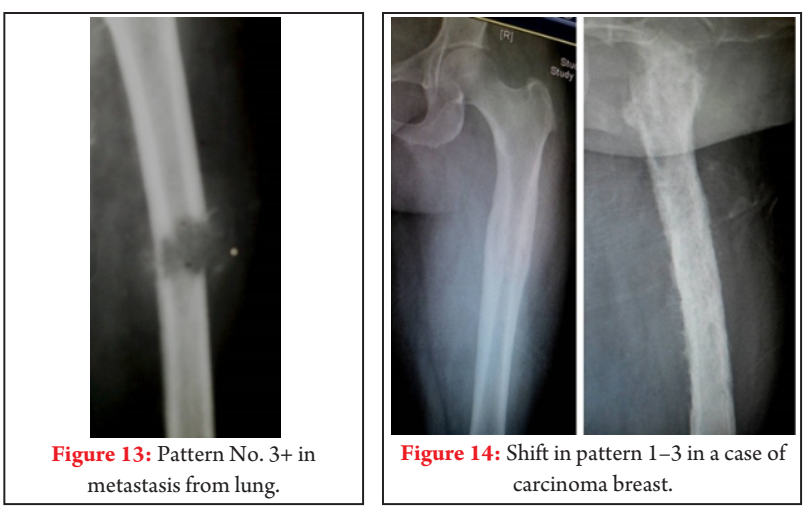

is its own cell that had turned malignant. It is difficult to identify the enemy. All these aspects will be reflected in the X-ray. Margins of the lesion are ill defined. You just can't tell where the lesion begins or ends. Bone is trying to defend by periosteal reaction. However, the aggressive tumor easily breaks it and goes out into the soft tissue.

Fig. 12 shows X-ray of a 6-year-old boy. Margins are ill defined. There is interrupted lamellar periosteal reaction and soft-tissue extension, that is, Pattern No. 3. MRI report is osteomyelitis. The patient did not respond to antibiotics. Per operatively, on making a drill hole in the cortex, drop of pus came out. Culture showed no growth of organisms. Cytology and biopsy report were Ewing sarcoma. This is the limitation of X-ray, in fact of all imaging modalities. We can definitely say the Pattern is No. 3. However, it is not possible to say whether it is infection or tumor.

In metastatic bone tumor, situation is still worse. Here, the pluripotent stem cells that are to become osteoblast are made to osteoclasts and help tumor to grow. Fig. 13 shows a case of carcinoma lung, the strongest offender bone can ever have, leading to permeative destruction in no time. There is no periosteal reaction and there is no surrounding sclerosis indicating total failure of the bone to react. We may call this as Pattern No. $3+$.

\section{Shift in Pattern}

In Fig. 14a, there is solid periosteal reaction. It may be infection or tumor. Bone is having upper hand. Fig. 14b shows X-ray of the same patient after one month. Solid periosteal reaction became speculated. It shows body yields. It was a case of carcinoma breast. Looking back, in this case, there is shift from pattern 1-3.

Fig. 15a shows X-ray of a boy with features of osteomyelitis. Such a lesion abutting physis is typical of infection and is having a classical Pattern No 2. Fig. 15b shows X-ray of the same patient after a course of antibiotics. There is shift from Pattern No. 2 to 1.

Fig. 10 and 16 belong to the same patient. Fig. 10 is the X-ray taken 3 weeks after he presented with features of acute infection. Fig. 16 shows X-ray after 3 years. There is a shift from Pattern 3 to 2. This showed bacteria which had an upper hand earlier, came to a compromise with the bone later, and confined itself to a cavity (cave) and remained dormant for 3 years. The patient was treated by saucerization, as the patient presented with recurrence of pain. (Cave was demolished).

Shift in pattern classically occurs in osteosarcoma undergoing chemotherapy [8]. If the response to chemotherapy is good, there will be shift from Pattern 3 to 2 or even 1. In cases where there is no response to chemotherapy which is not uncommon, there will not be 
any shift. Instead, the unhindered progress of the aggressive lesion can be appreciated in X-ray.

In short, X-ray clearly depicts what happens in the bone in various conditions much more than any other investigations $[9,10]$.

Declaration of patient consent: The authors certify that they have obtained all appropriate patient consent forms. In the form, the patient has given his consent for his images and other clinical information to be reported in the Journal. The patient understands that his name and initials will not be published, and due efforts will be made to conceal his identity, but anonymity cannot be guaranteed.

\section{Conflict of interest: Nil Source of support: Nil}

\section{References}

1. Mandal AK, Chaudhary S. Text Book of Pathology. Synapse Books. 1st ed. New

Delhi, India: Avichal Publishing Company; 2010.p. 51, 113.

2. Kumar V, Abbas AK, Fausto N, AsterJC. Robbins and Cotran Pathological Basis of

Diseases. Amsterdam, Netherlands: Elsevier; 2010.p. 1205-35.

3. Enneking WF, Bertoni F, The staging system for benign and malignant tumours of the musculoskeletal system. In: Nadarajan M, editors. Article Written in Principles of Orthopedic Oncology; 1997.p. 112-20.

4. Shimose S, Sugita T, Kubo T, Matsuo T, Nobuto H, Ochi M. Differential diagnosis between osteomyelitis and bone tumors. Acta Radiol 2008;49:928-33.

5. Stacy GS, Kapur A. Mimics of bone and soft tissue neoplasms. Radiol Clin North
Am 2011;49:1261-86.

6. Dorman HD, CzerniakB. Bone Tumors. 1st ed. United States: Mosby; 1998.p. 22.

7. Puthur D. X-ray patterns common to infection and tumours. Kerala J Orthop 2014;27:73-7.

8. Malawer MM, Sugarbaker PH. Musculoskeletal Cancer Surgery: Treatment of Sarcomas and Allied Diseases. Berlin, Germany: Springer; 2001.p. 59, 60.

9. Sutton D. Text Book of Radiology and Imaging. 7th ed. United Kingdom: Churchill Livingstone; 2003.p. 1153-60.

10. Miwa S, Otsuka T. Practical use of imaging technique for management of bone and soft tissue tumors.J Orthop Sci 2017;22:391-400.

\section{How to Cite this Article}

Puthoor D | Analysis of X-ray patterns in infection and tumor. Journal of Bone and Soft Tissues Tumors | Journal of Bone and Soft Tissue Tumors | May- August $2021 ; 7(2): 5-8$. 